\title{
ARTICLE
}

\section{ActiWiz - optimizing your nuclide inventory at proton accelerators with a computer code}

\author{
Helmut Vincke and Christian Theis* \\ CERN, Geneva 23, 1211 Geneva, Switzerland
}

\begin{abstract}
When operating an accelerator one always faces unwanted, but inevitable beam losses. These result in activation of adjacent material, which in turn has an obvious impact on safety and handling constraints. One of the key parameters responsible for activation is the chemical composition of the material which often can be optimized in that respect. In order to facilitate this task also for non-expert users the ActiWiz software has been developed at CERN. Based on a large amount of generic FLUKA Monte Carlo simulations the software applies a specifically developed risk assessment model to provide support to decision makers especially during the design phase as well as common operational work in the domain of radiation protection.
\end{abstract}

Keywords: high-energy accelerator; activation; FLUKA; Monte Carlo; risk assessment

\section{Introduction}

When operating an accelerator one always faces unwanted, but inevitable beam losses and radioactivity. On closer inspection it becomes evident that the radiological hazard exhibited by activated accelerator equipment has a clear logistical, operational as well as potentially significant financial impact on any further operation or process that is to be conducted.

The main parameters determining the radionuclide production are of course the actual conditions of the beam loss, the location of the equipment as well as the chemical composition of the activated material. While the former are mostly beyond influence the latter are often a matter of choice between suitable materials that fulfill all required mechanical properties while keeping the radiological hazard to a justifiable minimum. This of course requires a quantification and assessment of this hazard, which is non-trivial due to the multitude of parameters and aspects (operational, legal, financial, etc.) that have to be taken into account.

In order to facilitate this task the ActiWiz software has been developed. The design of this software is in line with so called "expert-systems" that are commonly used to provide a first level of support to decision makers during the planning phase. The aim of the tool is to allow non-expert users to obtain comparisons of the radiological hazard for arbitrary materials in an easy, yet flexible way (see Figure 1). It should be emphasized that this tool neither presumes to replace a full Monte Carlo study by an expert to obtain absolute values of radiological quantities linked to activated materials nor can it replace an assessment for very specific cases that

*Corresponding author. Email: christian.theis@cern.ch cannot be generalized. For such investigations the exact parameters of the beam loss would be required, which are naturally unknown in the design phase. As ActiWiz will mainly be used during the design phase it needs to base its calculations on generic scenarios which have been carefully chosen to represent the most common use-cases in the environment of an accelerator.

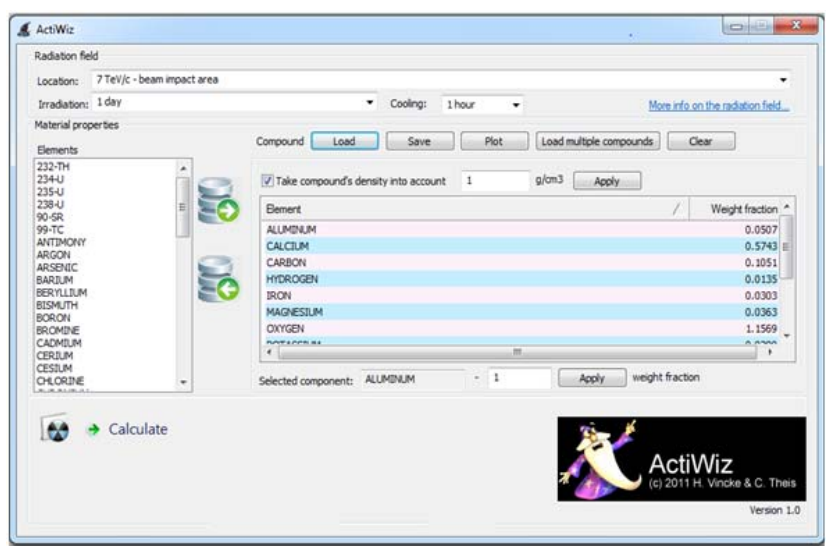

Figure 1. Screenshot of ActiWiz' non-expert mode. Based on a list of fundamental components (middle left) the user can define a compound (center) and calculate hazard factors for a defined radiation scenario (top).

\section{FLUKA simulations}

In order to assess the radiological hazard linked to the activation of arbitrary materials a two-step approach was adopted in order to produce the data, which serve as the basis for ActiWiz, with FLUKA [1,2]. The first step comprised the selection and calculation of particle fluence spectra (neutrons, protons and charged pions) at selected exemplary locations in accelerators for various 
energies of the primary proton beams. This selection of energies or momenta covers all accelerators currently in use at CERN. This comprises the Large Hadron Collider (LHC) with protons of $7 \mathrm{TeV}$, the Super Proton Synchrotron (SPS) with $400 \mathrm{GeV} / c$, the Proton Synchrotron (PS) with $14 \mathrm{GeV} / c$, the Booster with 1.4 $\mathrm{GeV}$, and the Linear Accelerator 4 (Linac4) with 160 $\mathrm{MeV}$. It should be noted that the mixture of kinetic energies and momenta in the labeling is due to historic reasons as those values became commonly accepted in the particle physics community for these specific accelerators. The maximum proton momentum of the PS is actually found to be $26 \mathrm{GeV} / c$. However, the particles mainly used in the machine are protons at $14 \mathrm{GeV} / c$ and thus, the major contribution to activation will stem from protons with the latter momentum. The corresponding reasoning has also been applied for choosing the energy/momenta also for the other accelerators in order to determine potential activation levels.

In a second step the calculated fluence spectra were used to determine the nuclide production vectors resulting from the exposure of a thin disk, made out of a pure chemical element, to the aforementioned radiation environments. These data were subsequently used for the actual assessment of the radiological hazard as described in the following sections. Extensive benchmarks of FLUKA with respect to the prediction of radionuclide production can be found in refs $[3,4]$.

\subsection{Determination of generic irradiation scenarios}

In order to systematically characterize potential activation levels a number of generic irradiation scenarios had to be defined and characterized in terms of their radiation environment. These scenarios should represent different locations where equipment is commonly installed. For this purpose the accelerator was implemented in FLUKA as a simplified tunnel structure with a concrete enclosure of $200 \mathrm{~cm}$ and a massive iron cylinder, acting as a stand-in for a magnet, or a thin iron cylinder representing a hypothetical target in the center of the tunnel structure. The target had a radius of $3 \mathrm{~cm}$ and a length of $100 \mathrm{~cm}$, whereas the dimensions of the magnet stand-in were adapted to resemble the actual magnet dimensions for each of the accelerators. For example for the LHC a bulky iron cylinder with a radius of $30 \mathrm{~cm}$ and a length of $200 \mathrm{~cm}$ was assumed whereas a Booster magnet was modeled with a radius of $50 \mathrm{~cm}$ and a length of $155 \mathrm{~cm}$.

The respective geometry was used to perform FLUKA calculations of particle fluence spectra at 7 pre-defined locations described in Table 1 and depicted in Figure 2, assuming the beam impact of protons either on the massive magnet stand-in or on the target. For a more detailed account of the scenarios please refer to [5]. For each accelerator the proton, neutron and charged pion fluence was determined at each of the locations defined in Table 1 as these particles are the main sources for activation at high-energy hadron accelerators. Subsequently, these spectra were used as source terms to determine the nuclide production using a second set of
FLUKA Monte Carlo simulations. For this purpose a custom routine was implemented in FLUKA which allowed for simulating a mixed radiation environment as a source term, using a probability mixing sampling technique. To this radiation field disks of one mm thickness were exposed and the nuclide production was recorded. Each of these disks was assumed to be made out of one of 69 fundamental components (63 stable chemical elements and 6 isotopes), which could be treated by FLUKA at that time (development version Dec. 2010). Clearly, disks of one mm thickness cannot account for significant self-shielding. At first glance this might be considered as a draw-back. However, this effect can only be taken into account fully if clearly defined dimensions of a piece of equipment and detailed information on the beam-loss are available. On one hand this is usually not the case during the design phase and on the other hand specific Monte Carlo studies are required for this purpose.

Table 1. Description of the locations in a hypothetical accelerator tunnel for which the radiation environment was characterized. The particle fluence spectra following a hypothetical beam loss were characterized for these positions considering all of CERN's accelerators ranging from the Linac4 to the LHC.

\begin{tabular}{ll}
\hline ID & Characterized radiation environment \\
\hline 1 & $\begin{array}{l}\text { beam impact area } \\
\text { within bulky material (e.g. magnet) } \\
\text { surrounding the beam impact area } \\
\text { adjacent to bulky material surrounding } \\
\text { the beam impact area } \\
3\end{array}$ \\
4 & $\begin{array}{l}\text { close to concrete tunnel wall (loss on } \\
\text { bulky object) } \\
\text { behind massive concrete shielding }\end{array}$ \\
6 & $\begin{array}{l}10 \text { cm lateral distance to a target } \\
\text { close to concrete tunnel wall (loss on } \\
\text { target) }\end{array}$ \\
\hline
\end{tabular}

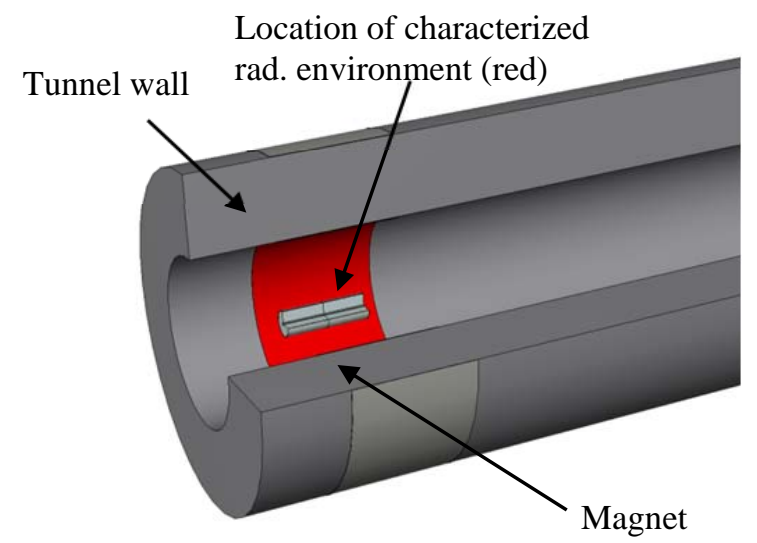

Figure 2. Illustration of the simplified accelerator tunnel geometry implemented in FLUKA in order to characterize the radiation environment at different locations. The red surface depicts the volume close to the tunnel wall that was studied as an exemplary radiation environment (ID 4 from Table 1). 


\section{Risk assessment model}

In order to quantify a radiological hazard which is linked to a material it is necessary to define a measure that eventually reflects the risk. This seemingly easy undertaking turns out to be rather complex because the associated risk depends on a large set of different parameters and use-cases of a material. For example, in operational radiation protection some materials, which exhibit significant dose-rate levels shortly after the irradiation, can pose a problem if access to the accelerator is required quickly. However, if the radioactivity decreases significantly within a period of a few years due to decay, then the material might not represent a hazardous object from the point of view of radioactive waste, for which storage and cooling times of several years are not uncommon. Consequently, different cooling scenarios have to be considered for the radioactive waste aspect of materials.

In addition it should be noted that there is another conceptual difference that has to be taken into account. In contrast to the operational hazard, which at CERN is mainly represented by external irradiation against which safety measures can be devised, the waste related hazard is linked to the so-called clearance concept and as such to exemption levels (LE values), which in Switzerland are defined in terms of activity $(\mathrm{Bq})$ or specific activity $(\mathrm{Bq} / \mathrm{kg})[6]$. Convolving the nuclide inventory with these limits yields a factor, which if greater than 1 classifies material as radioactive. Once the activity levels drop and the convolution would yield a value smaller than 1, the material can be regarded as non-radioactive and can be treated and disposed of as conventional material which is beyond regulatory control. Therefore, two distinct risk factors need to be assessed of which one corresponds to the operational risks and the other to waste related hazards.

\subsection{Quantification of the radiological hazard}

As a result of the FLUKA calculations for each element a nuclide vector is obtained which contains all nuclides produced in the target element $\boldsymbol{N}=\left\{N_{1}, \ldots, N_{i}\right\}$. This nuclide vector is a function of the radiation environment which is parameterized by the energy of the primary particles and the spatial location of the target element in the accelerator $N=N(E, \vec{x})$. For each scenario a set of different irradiation periods $\tau_{i}$ as well as cooling periods $\tau_{c}$ has been defined and the resulting specific activity $A=A\left(E, \vec{x}, \tau_{i}, \tau_{c}\right)$ has been calculated with FLUKA. The irradiation periods chosen consisted of a set comprising 1 second, 1 day, 1 week, 200 days (= average yearly runtime of the LHC) and 20 years. As such, various cases from an instantaneous beam loss up to the anticipated average life-time of a machine are covered. Of course the real runtime of an accelerator might exceed the time span of 20 years depending on the respective needs and physics programs. However, after such a long period most isotopes can be expected to have reached saturation levels and therefore, longer runtimes should be of minor influence in general. In addition it should be noted that comparisons of the hazards of a given set of materials are done on a relative and not on an absolute basis.

The cooling periods were selected to allow for the investigation of the impact of rather short lived isotopes on risk factors after a time span of 1 hour, as well as the evaluation of risk factors after typical waiting times before a technical stop or the yearly accelerator shutdown. These times extend from several hours to months but for the assessment of radioactive waste notably longer cool-down periods of several years have been included as well. The set comprised $1 \mathrm{~h}, 6 \mathrm{~h}, 1 \mathrm{~d}$, $1 \mathrm{w}, 1 \mathrm{~m}, 4 \mathrm{~m}, 2 \mathrm{y}, 5 \mathrm{y}, 10 \mathrm{y}$ and $20 \mathrm{y}$.

As a result of this calculation an extensive nuclide vector is obtained for each of the 69 fundamental components. However, the nuclide vector by itself is not suitable to describe the hazard because high activity levels do not necessarily lead directly to severe radiological consequences. Therefore, each nuclide inventory was subsequently convolved either with activity-to-dose conversion functions to obtain an operational hazard factor, or exemption limits (LE) to determine a waste related hazard factor [6]. In order to calculate the respective hazard factor for a compound consisting of several chemical elements, the results of each element are weighted with the respective weight fraction representing the abundance of the element in the compound and subsequently summed up. This yields an operational hazard factor $\lambda_{\text {op }}$, reflecting expected dose rate levels, and a waste related factor $\lambda_{\text {waste }}$ which signals if a material would be considered as radioactive or as conventional material. However, these quantities depend on a number of parameters like accelerator energy $E$, location $\vec{x}$, irradiation $\tau_{i}$ and cooling period $\tau_{c}$ which result in several thousand hazard values for a given material.

This high level of diversification is clearly too complex to form a viable basis for the selection process of materials. Therefore, some effort has been made to reduce this complexity by further generalization with respect to cooling as well as irradiation periods leading to the introduction of so-called global hazard factors.

\subsubsection{Global hazard factors}

While the selection of accelerator energy, location and irradiation period can be left to the end-user, the situation is more difficult and subtle with respect to cooling periods which strongly influence the actual radiological hazard. In order to obtain easily comparable assessments it was decided to remove this degree of freedom by directly including information on this parameter in the risk assessment model.

As mentioned before the operational hazard is related to the dose rate. However, strictly speaking this does not reflect an actual risk because a risk is not only correlated to the degree of impact but also to the probability of occurrence. Therefore, the global operational hazard factors are defined as the weighted sum of the dose-rates encountered for all considered cooling periods $\mathrm{N}_{\mathrm{c}}$. The 
weights $\xi_{t c}$ are chosen to represent the average intervention time of personnel in radioactive areas after the respective cooling time. As a consequence the global operational hazard factor for a compound $\left(\mathrm{G}_{\mathrm{op}}\right)$ is calculated as a sum of dose-rates multiplied with a corresponding average intervention time. The resulting value can be understood as a measure of the anticipated total dose for all cooling scenarios:

$$
G_{\boldsymbol{o p}}\left(\mathrm{E}, \overrightarrow{\mathrm{x}}, \tau_{i}\right)=\sum_{t_{c}=1 \text { hour }}^{N_{t c}}\left(\sum_{k=1}^{M}\left(\mu_{k, H *(10)}\left(\tau_{i}, \tau_{c}\right) * \frac{\omega_{k}}{\sum_{j} \omega_{j}}\right)\right) * \xi_{t c}
$$

with $\mu_{\mathrm{k}, \mathrm{H}^{*}(10)}$ denoting the result of the convolution of the produced isotopes with the activity-to-dose conversion factors, $\mathrm{k}$ being the index of the chemical element, $\mathrm{M}$ representing the number of constituents of the compound and $\omega_{k} / \sum_{j} \omega_{j}$ indicating the weight fraction of the chemical element $\mathrm{k}$. The importances $\xi_{t c}$ applied to the single operational hazard factors were derived from operational experience with interventions carried out in the SPS [5]. These importances reflect the average intervention time per intervention multiplied with the average number of interventions per year as a function of cooling time. Naturally these weights would have to be adapted for other facilities, for example hadron therapy centers, which have significantly different maintenance and shut-down patterns compared to particle research centers like CERN. However, the risk assessment model by itself is not restricted to high energy proton accelerators.

As can be seen from Equation (1) the metric represented by the global hazard factor $G_{\text {op }}$ only depends on the accelerator energy, the location and the irradiation period. For the corresponding waste related global hazard factor $G_{\text {waste }}$ only one cooling time of 20 years has been considered as it is intended to store the material on-site as long as possible to reduce the amount of material that has to be disposed of as radioactive waste:

$$
G_{\text {waste }}\left(\mathrm{E}, \overrightarrow{\mathrm{x}}, \tau_{i}\right)=\sum_{k=1}^{M}\left(\mu_{k, L E}\left(\tau_{i}, \tau_{c}\right) * \frac{\omega_{k}}{\sum_{j} \omega_{j}}\right)
$$

with $\mu_{\mathrm{k}, \mathrm{LE}}$ denoting the result of the convolution of the produced isotopes, originating from the chemical element k, with the Swiss exemption limits (LE) [6].

Since the operational as well as the waste related global hazard factors represent two different hazard measures, the former being related to total dose whereas the latter being related to costs, they both need to be normalized in order to obtain a comparable metric. In contrast to a comparison for only one specific and well-defined irradiation \& cooling scenario the choice of reference to be used for the normalization of integral values covering multiple cooling periods bears some intricate difficulties.

The range of hazards is defined by the minimum and maximum hazard stemming from the 69 studied fundamental components. No compound can have a smaller or bigger global hazard than the fundamental constituent with the minimum and maximum hazard respectively. Hence, an adequate reference can be defined by choosing one member of the aforementioned set for normalization. As a first idea the average hazard of the 69 fundamental components was chosen as the reference value. However, as a consequence of its mathematical definition the average is prone to be influenced significantly by outliers which introduce a bias. In case of the existence of a fundamental component with an exceptionally high hazard (e.g. ${ }^{235} \mathrm{U}$ ) this would lead to a very high reference value used for normalization. As a consequence the ratio of all materials, which do not contain this one highly critical contributor, would result in seemingly low hazard values.

A better choice for an adequate reference can be found in the fundamental component defined by the median of the hazard classification. In probability theory the median is described as the numerical value separating the upper half of a population from the lower half. By using the median hazard of the 69 fundamental components as the reference value for defining the normalized global factors, an undesirable bias can be avoided. Eventually the normalized values obtained for the risk assessment reflect how the radiological risk of a certain compound relates to the median risk represented by using equipment made out of the median element in one of the studied radiation environments.

Figure 3 illustrates the normalized global operational hazard factor based on equation (1), comparing stainless steel 304L with $0.1 \mathrm{wt} \%$ of Cobalt, pure iron and aluminium. 20 year of irradiation next to a magnet in the LHC have been assumed and it can be seen that the same object made out of aluminium would exhibit an operational hazard that is about 6 times lower than iron or 11 times lower than steel 304L.

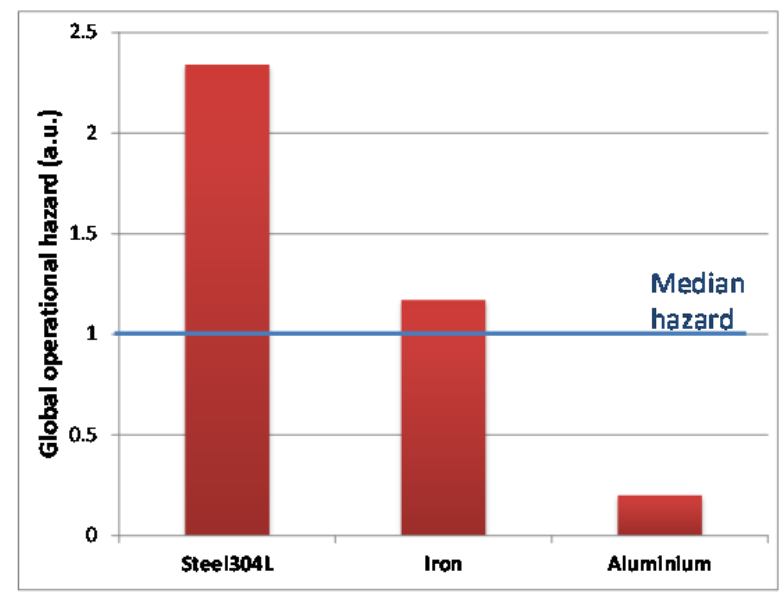

Figure 3. Comparison of the global operational hazard, based on residual dose rates, of steel 304L ( $0.1 \mathrm{wt} \%$ of Cobalt), pure iron and aluminium located next to a magnet in the LHC. 20 years of irradiation have been assumed. 


\section{Summary and conclusions}

In order to provide assistance for the selection of materials for particle accelerators at CERN a tool (ActiWiz) has been developed to assess the radiological risk of arbitrary materials. For this purpose an extensive Monte Carlo study using the FLUKA Monte Carlo code to assess the nuclide production of 69 fundamental components for 35 different generic radiation environments (7 locations for each of the 5 main energies used in CERN's accelerators) has been carried out. The data form the basis for a specifically developed risk assessment model which has been implemented in the ActiWiz computer code. This allows the user to easily define compounds out of the 69 studied fundamental constituents and compare their radiological risks in terms of external irradiation and in terms of the probability of creating radioactive waste. The former being denoted by the global operational hazard factor and the latter being indicated by the global waste hazard factor. Due to the graphical user interface the user can quickly define compounds and evaluate the associated risks within a few seconds on today's computer hardware. The code has already been used to create an extensive hazard classification for the most common materials used in CERN's accelerators.

Future extensions of the tool envisage the inclusion of additional radiation environments covering specifically the LHC detectors.

\section{Acknowledgements}

The authors wish to thank Doris Forkel-Wirth, Nadine Conan, Stefan Roesler and Ralf Trant for valuable discussions and their feedback. We would also like to acknowledge Robert Froeschl for kindly providing the activation data for Zinc and Matthias Karacson for reviewing the manuscript. In addition we would also like to express our gratitude to Steve Myers for supporting and promoting this project.

\section{References}

[1] A. Fassò, A. Ferrari, J. Ranft and P.R. Sala, FLUKA: A Multi-Particle Transport Code, CERN Yellow Report, INFN/TC_05/11, SLAC-R-773, (2005).

[2] G. Battistoni, S. Muraro, P.R. Sala, F. Cerutti, A. Ferrari, S. Roesler, A. Fassò and J. Ranft, The FLUKA code: Description and benchmarking, Proceedings of the Hadronic Shower Simulation Workshop 2006, Fermilab 6-8 September 2006, M. Albrow and R. Raja eds., AIP Conf. Proc., 896, (2007) pp. 31-49.

[3] M. Brugger, H. Khater, S. Mayer, A. Prinz, S. Roesler, L. Ulrici and Hz. Vincke, Benchmark studies of induced radioactivity produced in LHC materials, Part 1: specific activities, Rad. Prot. Dosim. 116 (2005), pp. 6-11

[4] M. Brugger, A. Ferrari, S. Roesler and L. Ulrici, Validation of the FLUKA Monte Carlo code for predicting induced radioactivity at high-energy accelerators, Nucl. Instrum. Meth. A562 (2006), pp. 827-829.

[5] H. Vincke and C. Theis, ActiWiz - A Computer Code to Model and Assess Radiological Hazards of Activated Material, CERN Technical Note CERN-DGS-2011-067-RP-TN, CERN, (2011).

[6] Bundesamt fuer Gesundheit, Strahlenschutzverordnung (StSV) 814.501 vom 22. Juni 1994 - Stand am 1. Januar 2012, (2012), Appendix 3. 